\title{
Flying Boresight Source for \\ Improved Testing and Calibration of Tracking Antennas and Advanced Flight Path Simulations
}

\author{
Dennis Haefner $^{1}$, Andreas Kimpe ${ }^{1}$, Ludwig Altenbuchner ${ }^{1}$, Peter Turner ${ }^{1}$ \\ 1 DLR Mobile Rocket Base, Oberpfaffenhofen, 82234 Wessling, Germany, \\ Dennis.Haefner@dlr.de
}

\begin{abstract}
:
The application of ground-based boresight sources for calibration and testing of tracking antennas usually entails various difficulties, mostly due to unwanted ground effects. To avoid this problem, DLR MORABA developed a small, lightweight, frequency-adjustable S-band boresight source, mounted on a small remote-controlled multirotor aircraft. Highly accurate GPS-supported, position and altitude control functions allow both, very steady positioning of the aircraft in mid-air, and precise waypointbased, semi-autonomous flights. In contrast to fixed near-ground boresight sources this flying setup enables us to avoid obstructions in the Fresnel zone between source and antenna. Further, it minimizes ground reflections or other multipath effects which affect antenna calibrations. In addition, the large operating range of a flying boresight simplifies measurements in the far field of the antenna and permits undisturbed antenna pattern tests. A unique application is the realistic simulation of sophisticated flight paths, including overhead tracking and unusual trajectories of fast objects such as sounding rockets. Likewise, dynamic tracking tests are feasible which provide crucial information about the antenna pedestal performance, particularly at high elevations, and reveal weaknesses in the autotrack control loop.
\end{abstract}

Key words: MORABA, flying, boresight, calibration, tracking antenna

\section{Introduction to MORABA}

The Mobile Rocket Base (MORABA) is a division of the German Aerospace Center (DLR), located in Oberpfaffenhofen, Germany. Since the 1960s, MORABA performs design, construction, preparation, and launch of sounding rockets and stratospheric research balloons, providing the scientific community a platform for research in atmospheric physics, microgravity, astronomy, materials science, and hypersonic technologies.

The MORABA group "Mobile Infrastructure", is in charge of conception, setup, and operation of mobile TT\&C (Telemetry, Tracking \& Command) ground stations required for sounding rocket and balloon missions. A new mobile telemetry station was purchased and set up recently. It comprises a main S-band tracking antenna system with a five-meter dish and a smaller secondary tracking antenna with a diameter of 1.5 meters. To qualify these new antenna systems, an extensive acceptance test was performed, which required a flexible and dynamic tracking target, in addition to a customary, mast-mounted boresight source.
Hence, the idea of a small and easily manageable airborne boresight was developed and successfully applied.

\section{Basic Idea of a Flying Boresight}

Stationary boresights installed on masts are the standard tool for qualifying, calibrating, and adjusting tracking antennas. Naturally, due to the proximity of the transmitter to ground, measurements based on mast-mounted boresights are affected by ground reflections, obstacles in the Fresnel zone between transmitter and antenna, and potential multipath effects from large nearby objects.

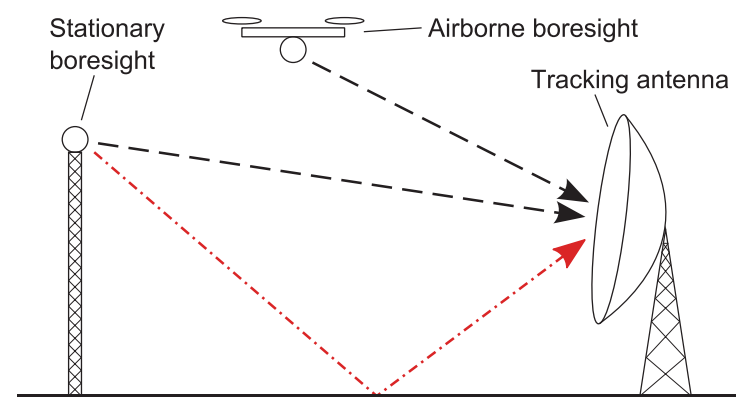

Fig. 1. Illustrating beam paths in-between tracking antenna and stationary / airborne boresight 
To overcome such disturbances and study dynamic tracking characteristics of an antenna, one option is to attach a transmitter to an airplane or helicopter, which then serves as a "flying boresight" (see Fig. 1).

Apart from avoiding ground effects and validating the tracking of moving targets, a flying boresight facilitates testing of critical antenna operating modes such as zenith passes or over-the-shoulder tracking. In addition, far field characteristics of an antenna are easier to evaluate with an aircraft, since - due to natural obstacles on ground - large distances between transmitter and antenna demand higher boresight elevation, and thus more effort in boresight placement.

In most cases, however, boresight tests with manned aircrafts require detailed preceding planning, formal authorization, and a considerable budget. Furthermore, essential antenna adjustment and calibration procedures, such as measurements of lobe geometry, antenna patterns, or autotrack error signals, demand an absolutely stationary positioned boresight. A GPS-supported helicopter could suffice this requirement. However, helicopters generally are not well suited tracking targets, due to signal reflections from the large and fast moving rotor blades that induce modulation in the signal amplitude, influencing the AM-based target tracking mechanism of the antenna system.

\section{Multirotor UAV as Flying Boresight}

For the acceptance tests of their new tracking antenna system, MORABA developed a compact, yet versatile flying boresight system based on a remote-controlled multirotor unmanned aerial vehicle (UAV). This concept combines advantages of fixed-wing and hover-capable aircrafts, and coalesces desirable attributes such as compactness, flexibility, ease of use, and low costs.

\section{UAV Platform}

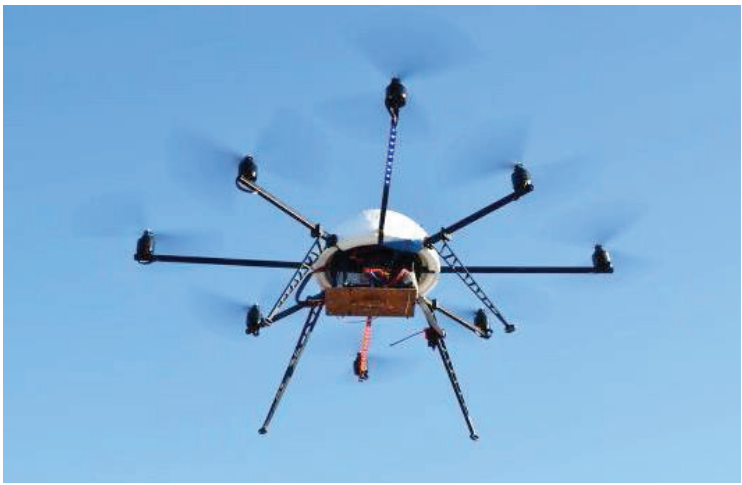

Fig. 2. MORABA's Flying Boresight in mid-air
The first prototype of the Flying Boresight was built using a commercially available rotorcraft UAV, propelled by eight electric motors (Fig. 2). It has a diameter of about one meter and a takeoff weight of approximately 2.5 kilograms. The UAV is equipped with a microcontrollerbased flight attitude control and navigation system comprising a precision GPS receiver, barometric altitude sensor, magnetic compass, and three-axis accelerometers and gyros. Positionhold and altitude-hold modes enable exceedingly easy handling of the UAV in flight. Hence, all necessary skills can be acquired in a minimum of time. Without intervention of the remote-controlling pilot, the UAV stays "fixed" in mid-air, even during moderate wind gusts. Likewise, autonomous flights along trajectories based on GPS waypoints are possible. The pilot is supported by a built-in telemetry link of the remote control $(\mathrm{RC})$ system, which provides all relevant real-time flight data of the UAV. These include position, altitude, orientation, ground range, power consumption, battery status, and link quality. Even more detailed information is available using a separate ground station software, displaying the flight path and enabling in-flight waypoint planning.

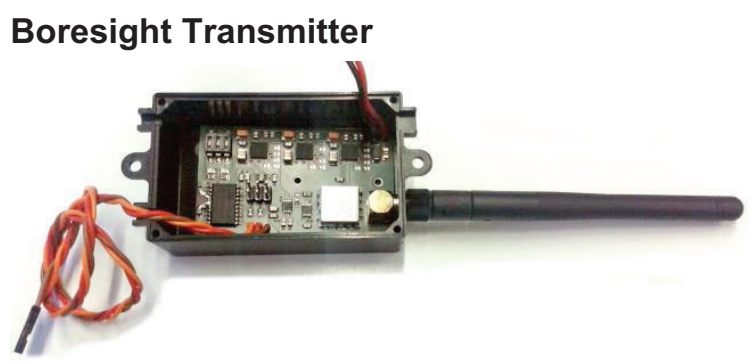

Fig. 3. Miniature S-band boresight transmitter with rod antenna (for testing only, later replaced by actual rocket compliant antenna)

Since conventional boresight transmitters are too large and heavy to be lifted by small UAVs, MORABA designed a simple, ultra-small and lightweight S-band transmitter (see Fig. 3), tunable between 2.1 and $2.4 \mathrm{GHz}$ via a standard channel of the UAV RC link. A microcontroller decodes the RC signal and adjusts a voltagecontrolled oscillator (VCO) through a precision digital-to-analog converter (DAC). Due to the required frequency stability in the order of few $\mathrm{kHz}$ and the VCO's sensitivity to temperature changes, the circuit is optimized for minimal temperature dependence and is placed in a thermally isolated box.

\section{Advantages of a UAV as Flying Boresight}

Compared to conventional airplanes or helicopters, MORABA's UAV-based Flying Boresight has the following unique features: 
- Its intrinsic ability to precisely hold a position in mid-air replaces unfeasibly tall boresight masts. High elevation is essential for pattern tests, lobe and error signal measurements free from ground effects.

- Due to low weight and very dynamic flight characteristics, complex flight patterns of fast tracking targets can be imitated.

- Trajectories can be exactly repeated, either pre-programmed or by recording a manual flight. This simplifies repetitive tests and failure analysis.

- The RC pilot can stay close to the antenna control unit (ACU). Therefore, only minimal pre-flight briefing is necessary, since flight maneuvers can be adapted spontaneously through immediate feedback from the ACU operator.

- No long-term preparation or planning is required. Apart from charging batteries and obtaining a flight permission, if any, the UAV is ready to fly within minutes.

- It is always available and immediately operational at any time. Due to its compactness and useful attributes, the Flying Boresight now is a standard component of the MORABA antenna equipment.

- In light of expenditure for conventional antenna tests with manned aircrafts, MORABA's Flying Boresight is unrivaled and has virtually no operational costs.

\section{Experiences with the Flying Boresight}

Mainly developed for acceptance tests of MORABA's new tracking antennas (Fig. 4), the Flying Boresight surpassed all expectations regarding usability, efficiency, and precision. Hence, it became an integral part of the standard antenna setup and calibration procedures.

During acceptance tests a manned aircraft was never needed, since all tracking tests were carried out by the Flying Boresight.

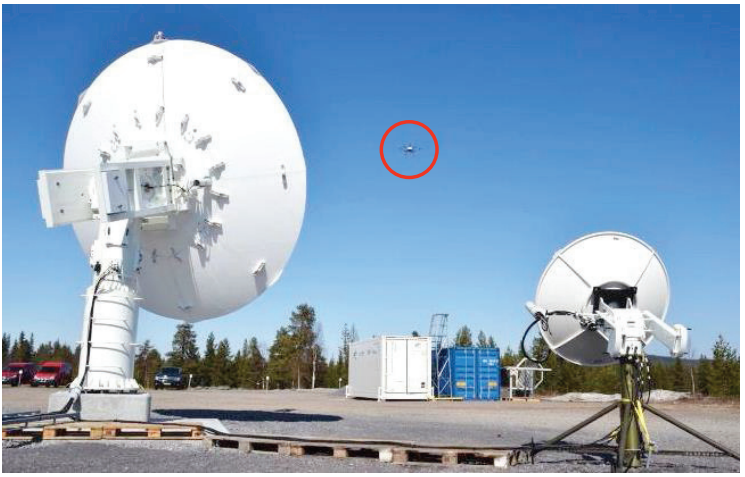

Fig. 4. Both MORABA antennas autotracking the Flying Boresight
Being delicate tracking targets due to high accelerations and elevations close to 90 degrees, sounding rockets impose stringent requirements on tracking antennas with regard to tracking speed and high-zenith tracking performance. Using the Flying Boresight, projected tracking speeds of up to $30 \%$ s and antenna pedestal accelerations of $30 \% \mathrm{~s}^{2}$ have been verified successfully. Likewise, the usually delicate gain increase within the azimuth control loop at high elevations - originating from conversion of feed azimuth to pedestal azimuth angles - was examined extensively with repetitive high-zenith passes. Only with the highly dynamic flight capabilities of the Flying Boresight was it possible to reveal oscillation tendencies of the antenna pedestal at high elevations. Comparatively inert flight characteristics of conventional aircrafts could have never triggered these deficiencies in the antenna pedestal control.

Thanks to the simple repeatability of critical flight patterns, the antenna manufacturer was able to tune the antenna pedestal control loop parameters in unprecedented detail, eliminating all oscillations while preserving maximum tracking responsiveness.

\section{Outlook}

Having proven excellent usability, MORABA continues research and development of its Flying Boresight. Among other optimizations, key improvements entail:

- Employing a more advanced UAV platform, which provides even better flight characteristics, higher payload capacity, and a collapsible frame for improved transportability

- As a consequence thereof will be a significant increase in flight time from about 14 minutes to roughly 40 minutes, for longer continuous measurements

- Replacement of the simple boresight transmitter with a full-featured, yet compact and lightweight telemetry simulation system for profound, real-scenario telemetry tests

- Upgrade to a professional grade digital RC system in the $900 \mathrm{MHz}$ ISM band

With these improvements, MORABA's Flying Boresight will be able to provide a comprehensive and versatile measurement and calibration tool for tracking antennas at a fraction of the cost of current methods, yet offering unprecedented usability and flexibility. 